\title{
Community Newsletter: Parental perceptions of autism over time, a new therapy for anxiety in autistic people
}

\author{
BY CHELSEY B. COOMBS
}

22 AUGUST 2021

Hello, and welcome to this week's Community Newsletter! I'm your host, Chelsey B. Coombs, Spectrum's engagement editor.

Parents' perceptions of autism have changed as the condition's prevalence has increased, according to a new paper that analyzed survey data over a 15-year period. Those with autistic children perceived their children's impairment as 23 percent greater at the end of the study period than the parents at the start did. And the perceived impairment increase was as high as 65 percent among parents with children whose autism traits were insufficient for a diagnosis.

"Our results clearly show that perceived impairment has increased with calendar year at any given [autism] symptom level, but even more so in individuals who previously would have been considered subsyndromal or 'only' exhibiting autistic traits," the researchers write.

This shift is due to the change over time in how autism is defined, they add - a point reiterated in a tweet from Uta Frith, emeritus professor of cognitive development at University College London in the United Kingdom.

https://twitter.com/utafrith/status/1425756863103180803

Frith wrote in depth about this topic for her July Autism Research Letter to the Editor, spurred on by Laurent Mottron's commentary on the same subject. Mottron, professor of psychiatry at the University of Montreal in Canada, will speak more on this subject in our August 31 webinar.

Tony Charman, professor of clinical child psychology at King's College London in the U.K., tweeted that the study was an "interesting look at secular changes over time." 


\section{Spectrum | Autism Research News}

https://www.spectrumnews.org

https://twitter.com/TonyASDorAFC/status/1425705735426646020

Our next thread comes from Lisa Quadt, a postdoctoral research fellow at the University of Sussex in the U.K. Quadt discusses her study on a new therapy to help target anxiety in autistic adults. Some 53 percent of autistic people meet the diagnostic criteria for an anxiety disorder at some point during their lives, whereas only 10 to 15 percent of non-autistic people do.

https://twitter.com/LisaQuadt/status/1424642239205150720

She and her colleagues tested a therapy called Aligning Dimensions of Interoceptive Experience, or ADIE. Previous research has shown that people with high anxiety levels are more sensitive to internal changes in their bodies, such as a change in heartbeat. And autistic people are more likely than non-autistic people to have trouble accurately measuring and describing bodily sensations, an ability called interoception.

ADIE involves giving feedback to people on how well they are measuring their own heartbeat, to help them increase their accuracy. The team's hypothesis - that being better able to understand and regulate interoceptive experience could lower anxiety - proved correct: Autistic people who went through six sessions of ADIE had less anxiety three months after treatment than those who went through a control therapy.

"We hope that ADIE can be incorporated into clinical practice as one tool against symptoms of anxiety in autistic and non-autistic adults," Quadt tweeted.

https://twitter.com/LisaQuadt/status/1424642242778632193

Hakwan Lau, team leader of the Consciousness Lab at the RIKEN Center for Brain Science in Saitama, Japan, tweeted that ADIE "looks like it could be useful for e.g. alexithymia, or just folks with anxiety disorders in general."

https://twitter.com/hakwanlau/status/1424790598205149186

Quadt responded that ADIE produced similar results in non-autistic people and that a study was underway to understand its effects on people with anxiety disorder and joint hypermobility.

https://twitter.com/LisaQuadt/status/1424805398683570182

And finally, David Mandell, professor of psychiatry at the University of Pennsylvania and Autism editor-in-chief, wins the award for 'tweet of the week,' in our opinion:

https://twitter.com/DSMandell/status/1427611770038931464 


\section{Spectrum | Autism Research News}

https://www.spectrumnews.org

Don't forget to register for our 31 August webinar with Laurent Mottron, professor of psychiatry at the University of Montreal in Canada, who plans to discuss "a radical change in our autism research strategy."

And on 28 September, Jeremy Veenstra-VanderWeele, professor of psychiatry at Columbia University, will speak about goals for developing new drugs for autism - and the barriers researchers may encounter.

That's it for this week's Community Newsletter from Spectrum! If you have any suggestions for interesting social posts you saw in the autism research sphere, feel free to send an email to me at chelsey@spectrumnews.org. See you next week!

Cite this article: https://doi.org/10.53053/NFQZ7576 